\title{
Comparison of computational chemistry methods for the discovery of quinone-based electroactive compounds for energy storage
}

Qi Zhang ${ }^{1,2,3}$, Abhishek Khetan ${ }^{1,2}$, Süleyman $\mathrm{Er}^{1,2 *}$

${ }^{1}$ DIFFER - Dutch Institute for Fundamental Energy Research, De Zaale 20, 5612 AJ Eindhoven, The Netherlands

${ }^{2}$ CCER - Center for Computational Energy Research, De Zaale 20, 5612 AJ Eindhoven, The Netherlands

${ }^{3}$ Department of Applied Physics, Eindhoven University of Technology, Eindhoven 5600 MB, The Netherlands.

*E-mail: s.er@differ.nl

Keywords: Computational chemistry; High-throughput screening; Density functional theory; Redox flow battery; Quinones; Energy storage

\begin{abstract}
High-throughput computational screening (HTCS) is an approach that can enable rational and timeefficient discovery of electroactive compounds. The effectiveness of HTCS is dependent on the accuracy and speed at which the performance descriptors can be estimated for possibly millions of candidate compounds. Here, a systematic evaluation of computational methods, including force field (FF), semi-empirical quantum mechanics (SEQM), density functional based tight binding (DFTB), and density functional theory (DFT), is performed on the basis of their accuracy in predicting the redox potentials of redox-active organic compounds. Geometry optimizations at lower level theories followed by single point energy (SPE) DFT calculations including an implicit solvation model are
\end{abstract}


found to offer equipollent accuracy as the higher level DFT methods, albeit at significantly lower computational costs. Effects of implicit solvation on molecular geometries and SPEs, and their overall effects on the prediction accuracy of redox potentials are analyzed in view of computational cost versus prediction accuracy, which outlines the best choice of methods corresponding to a desired level of accuracy. The modular computational approach presented here is expected to be applicable for accelerating virtual studies on functional quinones and the respective discovery of candidate compounds for energy storage.

\section{Introduction}

Commercial utilization of intermittent renewable energy sources, such as solar and wind, requires large-scale, low-cost, and durable energy storage technologies to balance the mismatch between the energy supply and demand. Redox flow batteries (RFBs) are recognized as prime candidates for largescale and long-term storage of electrical energy. ${ }^{1,2}$ RFBs have external storage tanks that store the liquid-phase redox-active electrolyte material separated from the electrochemical reaction cells. This unique design feature is advantageous as it decouples the battery's power and energy density scaling, while also facilitating easier maintenance and recycling. ${ }^{2}$

Conventional RFBs operate using metal-based electrolyte materials, such as vanadium, iron, zinc, lead and chromium. ${ }^{3}$ They, however, face technical challenges of ion crossover through the membranes and sluggish reaction kinetics. ${ }^{4}$ Additionally, high-costs and the risks associated with metal toxicity have hindered the widespread deployment of metal-based RFBs. ${ }^{5,6}$ RFBs employing organic redox active materials offer a promising alternative to metal-based electrolytes, since they can sustainably be sourced from earth-abundant elements and further modified structurally to tune their key batteryrelevant properties when necessary. ${ }^{1,2}$ The emerging classes of organic redox-active materials, which have been utilized in RFBs, consist of viologens, ${ }^{7,8}$ alloxazines,,${ }^{9,10}$ phenazines, ${ }^{11,12}$ nitroxide radicals ${ }^{13}$ and quinones. ${ }^{14-18}$ 
Among organic-based redox couples, quinone compounds are ubiquitous in nature. ${ }^{19}$ With their fast redox kinetics ${ }^{15,20}$ and tunable properties owing to their chemical diversity, ${ }^{1,21}$ they are increasingly being utilized as electroactive materials in new, advanced RFB technologies. Accordingly, a tremendous effort has been made in recent years to develop aqueous RFBs (ARFBs) that utilize quinones, such as the functionalized benzoquinones, ${ }^{22,23}$ naphthoquinones, ${ }^{20,22}$ and anthraquinones, ${ }^{22,24}$ as electroactive materials. Research has shown that these molecules undergo a coupled two-electron two-proton redox reaction in aqueous media. ${ }^{14}$ However, these molecules offer low energy densities in a practical ARFBs as they are not very soluble and their half-cell redox potential is not close enough to $0 \mathrm{~V}$ (vs SHE), which is desired for ARFB anolytes. ${ }^{22,25,26}$ Therefore, a major challenge for organic ARFBs is to tune the properties of the electroactive compounds to meet the practical requirements of high power and energy density.

To make an ARFB with large cell voltage, maximizing the redox potential window of quinone-based compounds is essential. Recent experimental and computational studies show that the redox potential of organic ARFBs can significantly be influenced by functionalizing them with electronwithdrawing/donating groups. ${ }^{1,2}$ Assary et al. ${ }^{27}$ and Aspuru-Guzik et al. ${ }^{28,29}$ used HTCS for creating virtual libraries of candidate electroactive compounds populated with the functionalized compounds of quinones and predicting their redox properties. These studies utilized robust quantum chemical calculations to estimate the thermodynamic properties of compounds and identify the most promising candidates for further study. Thus, they showed how hierarchical HTCS methods can be used to accelerate the predictions of key properties of redox-active molecules.

Using quantum chemical calculations to predict the redox properties is, however, a computationally expensive task. The approach is particularly not well-suited for HTCS studies on the enormous space of conceivable molecules. Therefore, it is necessary to determine the trade-offs between the accuracy and computational expense. While there has been a significant increase in the number of HTCS efforts in the area of RFBs, ${ }^{27-34}$ to the best of our knowledge, an analysis of the effect of factors, such as the 
level of theory for optimization of molecular geometry, inclusion/exclusion of solvation effects and the level of theory for the calculation of energy, on the prediction accuracy are not available in the current literature.

To address the issue, in this article, we systematically evaluate the performance of different computational methods, including DFT, DFTB, ${ }^{35}$ and SEQM ${ }^{36-38}$ We compare these methods based on their accuracy in predicting experimentally measured redox potentials of quinone based molecules from four different sources. Besides, we make first-order comparisons of the computational expense of these approaches and suggest the best approach that offers very good prediction accuracy at a much lower computational cost. The results provide new insights into the various factors that influence the accuracy of computational methods for predicting redox potentials. The new findings are expected to be useful for both customary and HTCS efforts that are aimed at the study of redox-active molecules that are of importance in bio- and electro-chemical conversion reactions also beyond the ARFBs.

\section{Methods}

\subsection{Thermodynamic Principle}

The main aim of this work is to develop a computational framework to quickly and accurately predict the redox potentials of quinone-based ARFB electrolyte compounds with high charge-to-weight ratio. During a redox reaction in the aqueous phase:

$\mathrm{Q}+2 \mathrm{H}^{+}+2 e^{-} \rightarrow \mathrm{QH}_{2}$

The hydroquinone, $\mathrm{QH}_{2}$, compounds can be generated from the quinone, $\mathrm{Q}$, compounds via a twoelectron two-proton redox reaction. ${ }^{14}$ A quantitative measure of the favorability of a given reaction is the change in standard Gibbs free energy, $\Delta G_{\mathrm{rxn}}^{\mathrm{o}}$. According to the Nernst equation, the equilibrium 
potential of a redox reaction, $E^{\circ}$, is related to the change in the standard Gibbs free energy per coulomb of charge transferred during the electrochemical reaction as:

$E^{\mathrm{o}}=-\Delta G_{\mathrm{rxn}}^{\mathrm{o}} / n F$

where $n=2$ is the number of electrons and $F$ is the Faraday constant. Typically, $E^{\mathrm{o}}$ is measured relative to the Standard Hydrogen Electrode (SHE) and $\Delta G_{\mathrm{rxn}}^{\mathrm{o}}$ is computed at standard temperature $T=298.15$ $\mathrm{K}$ and pressure $p=1 \mathrm{~atm}$. To calculate $\Delta G_{\mathrm{rxn}}^{\mathrm{o}}$ we use the following equation:

$\Delta G_{\mathrm{rxn}}^{\mathrm{o}}=G^{\mathrm{o}}\left(\mathrm{QH}_{2}\right)-\left[G^{\mathrm{o}}(\mathrm{Q})+G^{\mathrm{o}}\left(\mathrm{H}_{2}\right)\right]$

in which $\Delta G_{\mathrm{rxn}}^{\mathrm{o}}$ is expressed simply as the difference in the standard free energies of the reactants and products. Thermodynamically, $\Delta G_{\mathrm{rxn}}^{\mathrm{o}}$ can be described as a sum of contributions arising from the change in internal energy $\left(\Delta U_{\mathrm{rxn}}\right)$, pressure-volume $\left(p \Delta V_{\mathrm{rxn}}\right)$ and entropic $\left(T \Delta S_{\mathrm{rxn}}\right)$ contributions due to reaction as:

$\Delta G_{\mathrm{rxn}}^{\mathrm{o}}=\Delta U_{\mathrm{rxn}}+p \Delta V_{\mathrm{rxn}}-T \Delta S_{\mathrm{rxn}}$

The change in internal energy can be further decomposed as $\Delta U_{\mathrm{rxn}}=\Delta E_{\mathrm{rxn}}+\Delta \mathrm{ZPE}$, where $\Delta E_{\mathrm{rxn}}$ is simply the reaction energy and $\triangle \mathrm{ZPE}$ is the change in zero-point energy. In the present work, the zeropoint energy contributions to the internal energy, changes in pressure-volume and entropic contributions are neglected, thus effectively using the approximation:

$\Delta G_{\mathrm{rxn}}^{\mathrm{o}} \cong \Delta E_{\mathrm{rxn}}=E^{\mathrm{aq}}\left(\mathrm{QH}_{2}\right)-\left[E^{\mathrm{aq}}(\mathrm{Q})+E^{\mathrm{aq}}\left(\mathrm{H}_{2}\right)\right]$ 
where $E^{\text {aq }}$ represents the theoretically calculated internal energy of a species in the aqueous (aq) phase. All the other contributions are ignored because they require extra calculation steps, computational resources, and thus, are not suitable from the perspective of HTCS. The effects of ignoring these other contributions on the accuracy of predictions are discussed in Section 3.1. The descriptor of choice in this work, $\Delta E_{\mathrm{rxn}}$, is calculated with the inclusion of aqueous solvation effects, which requires additional computation time. In order to quantify the effect of solvation on prediction accuracy, another approximation is considered in this work by ignoring solvation such that Eq. (5) can be rewritten using internal energies calculated in the gas $(\mathrm{g})$ phase as:

$\Delta G_{\mathrm{rxn}}^{\mathrm{o}} \cong \Delta E_{\mathrm{rxn}}=E^{\mathrm{g}}\left(\mathrm{QH}_{2}\right)-\left[E^{\mathrm{g}}(\mathrm{Q})+E^{\mathrm{g}}\left(\mathrm{H}_{2}\right)\right]$

Under these set of approximations, the calculated change in internal energy $\Delta E_{\text {rxn }}$ from Eq. (5) and (6) can be linked to the measured redox potential using Eq. (2). In this work, we use various theoretical methods, as explained below in Section 2.2, to calculate $\Delta E_{\mathrm{rxn}}$, and discuss their performance for the prediction of experimentally measured redox potentials.

\subsection{Computational Workflow}

In this study, the MacroModel program is used for force field (FF) configurational searches and geometry optimizations, and Jaguar program ${ }^{39}$ is used for DFT calculations, all as implemented in the Materials Science Suite (version 2019-2) provided by Schrödinger, LLC. The MOPAC and DFTB calculations are performed using the ADF program. ${ }^{40}$ To make generalizable and consistent comparisons between various computational approaches, we developed a systematic workflow, as shown in Fig. 1. 


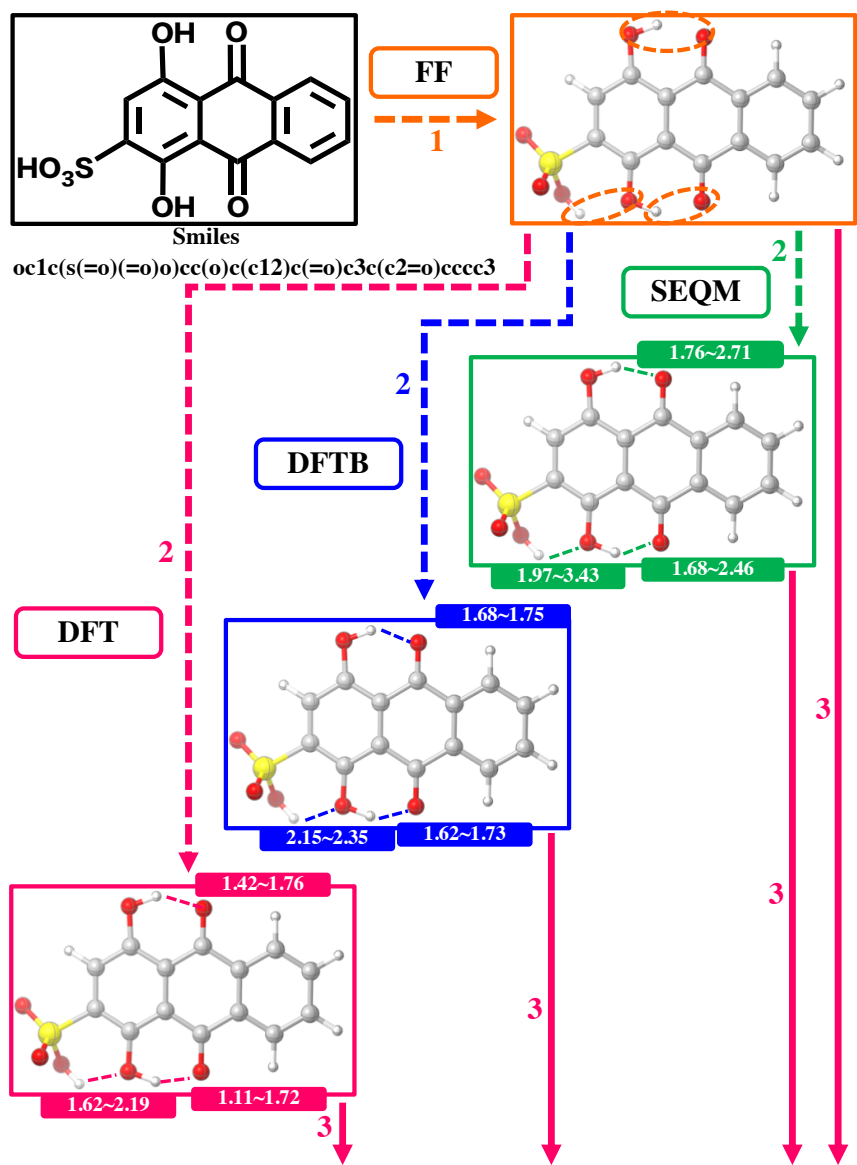

DFT calculated Single Point Energy (SPE) in gas-phase

$$
4:
$$

SPE in implicit Poisson Boltzmann solution-phase (PBF)

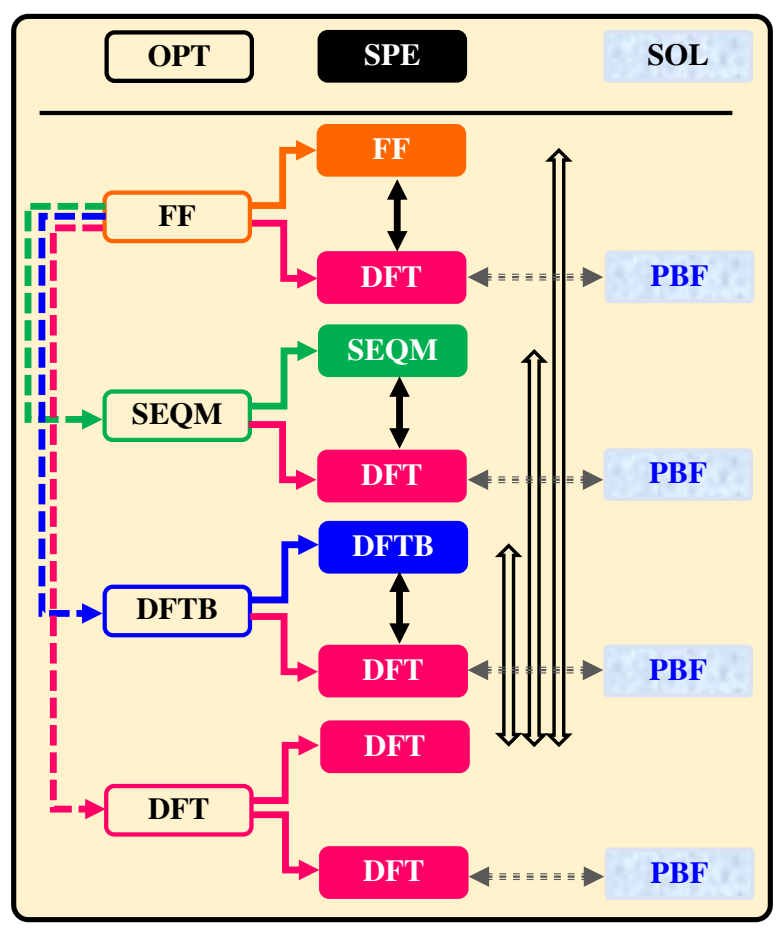

$\Delta$ [RMSE] in predictions of $E_{\exp }^{o}$ values between:

$\Uparrow \Delta^{t}$-gas phase energy and geometry at different levels of theory

$\uparrow \Delta^{\mathrm{e}}$ - energy computed at DFT level for a fixed geometry from a lower level theory

$\longleftarrow \equiv \equiv \equiv \Delta^{\mathrm{s}}-$ implicit solvation at DFT level

(a)

(b)

Fig. 1 (a) Schematic showing the chain of operations for geometry optimization (OPT) and single point energy (SPE) calculations at different levels of theory (b) A graphical summary of the various levels of approximations used for estimating $\Delta E_{\mathrm{rxn}}^{\mathrm{DFT}}$ in this work. The hollow black arrow with symbol $\Delta^{\mathrm{t}}$ represents the difference between gas phase energy and geometry at different levels of theory. The solid black arrow with symbol $\Delta^{\mathrm{e}}$ represents the difference between energy computed at DFT level for a fixed geometry from lower-level theory and energy computed at that given level of theory. The dotted gray arrow represents the solvation effect from gas-phase SPE to solution-phase SPE when the implicit solvation model is considered. For both (a) and (b), the text boxes with no background represent geometry optimization; the boxes with color background represent SPE calculations; the full boxes 
with water bubbles in the background represent solution phase SPE calculations using the implicit solvent model with water as the solvent.

Table 1. List of computational methods that are used for the optimization of geometries and the calculation of the single point energies of the molecules.

\begin{tabular}{l|l}
\hline FF & DFT \\
OPLS3e & LDA \\
\cline { 1 - 1 } SEQM & PBE \\
AM1 & PBE-D3 \\
MNDO & BLYP \\
MNDO & BLYP \\
PM3 & B3LYP \\
PM6 & B3LYP-D3 \\
PM6-D3 & PBE0 \\
PM6-D3H4X & PBE0-D3 \\
PM7 & HSE06 \\
RM1 & M08-HX \\
\cline { 1 - 1 } DFTB & \\
DFTB-D3 & \\
GFN1-XTB & \\
\hline
\end{tabular}

In this workflow, the starting point for any given molecule is its SMILES representation, ${ }^{41}$ which is a widely used form of graph-representation and can easily be generated for any given molecule. The SMILES representation is at first converted to a two-dimensional (2D) geometrical representation using a SMILES interpreter. Next,

(1) This 2D representation is converted to a three-dimensional (3D) geometry by applying the geometry optimization (OPT) scheme of OPLS3e $\mathrm{FF}^{42}$ to identify the lowest energy 3D conformer. It is important to note that in this workflow, the FF level geometry is the starting point for constructing all the considered approaches.

(2) The 3D geometry is further optimized in the gas phase at three different levels of theory, namely: SEQM, DFTB, and DFT. For SEQM and DFT, geometry optimizations are also carried out in 
the implicit aqueous phase, but these are not shown in Fig. 1 for the sake of simplicity. This step yields different 3D geometries and the corresponding SPE of the molecules.

(3) Next, SPEs of all the different 3D geometries are calculated using different DFT functionals. This step yields energy values that are directly comparable but are obtained from geometry optimizations that are performed at four different levels of theory.

(4) Finally, for the geometries obtained from the optimizations in the gas phase, the SPEs are recalculated, this time by including the effect of the aqueous medium (SOL) implicitly by using the Poisson-Boltzmann Solvation Model (PBF). ${ }^{39,43}$

The molecular structures of redox couples are optimized both in the gas and aqueous phases using the OPLS3e FF that provides a broad coverage of small compounds. ${ }^{42}$ The gas phase FF optimized geometries are used as inputs to perform gas and aqueous phase geometry optimizations using nine different SEQM methods (Table 1), including AM1, ${ }^{44} \mathrm{MNDO},{ }^{45} \mathrm{MNDOD},{ }^{46} \mathrm{PM} 3,{ }^{47} \mathrm{PM} 6,{ }^{48} \mathrm{PM} 6-$ D3, ${ }^{49} \mathrm{PM} 6-\mathrm{D} 3 \mathrm{H} 4 \mathrm{X},{ }^{38} \mathrm{PM} 7^{50}$ and RM1. ${ }^{51}$ The aqueous phase geometry optimizations at the SEQM level are performed using the COSMO-RS solvation model. ${ }^{52,53}$ The choice of this solvation method is constrained by the present availability in the ADF program. The gas phase FF optimized geometries are also used as inputs for DFTB level optimizations using the DFTB-D3 ${ }^{54}$ and GFN1-xTB ${ }^{55,56}$ methods. The DFTB-D3 computations are performed with a self-consistent charge cycle using the QuasiNANO-2015 parameter set, ${ }^{35}$ while the parameters for GFN1-xTB are taken from the work of Grimme et al..$^{55,56}$ The aqueous phase geometry optimizations of molecules are not performed with the DFTB method, since currently there is no available routine for this task in the ADF program. Finally, FF minimized geometries are used as inputs to perform geometry optimizations in the gas phase at the DFT level using the local density approximation (LDA), ${ }^{57}$ generalized gradient approximation (GGA), ${ }^{58}$ hybrid and meta-GGA functionals,${ }^{58}$ all of which vary drastically in their accounting of the exchange-correlation energy. A total of 11 functionals, also including some of the D3 dispersion ${ }^{59,60}$ corrected variants, are used for geometry optimizations and single-point energy calculations. As listed 
in Table 1, these functionals include LDA, ${ }^{57} \mathrm{PBE},{ }^{61,62} \mathrm{PBE}-\mathrm{D} 3,{ }^{59} \mathrm{BLYP},{ }^{63} \mathrm{BLYP}-\mathrm{D} 3,{ }^{59} \mathrm{~B} 3 \mathrm{LYP},{ }^{63,64}$ B3LYP-D3, ${ }^{59}$ PBE0,${ }^{65}$ PBE0-D3, ${ }^{59}$ HSE06, ${ }^{66}$ and M08-HX. ${ }^{67}$ Owing to their significantly higher computational expense, the DFT aqueous phase geometry optimizations are performed only with the following functionals: PBE, B3LYP, and M08-HX. The choice of these 3 functionals was motivated by the fact that they lie on three different rungs of the so-called Jacob's ladder of accuracy, ${ }^{58}$ namelyGGA, hybrid and meta-GGA. It was assumed that functionals at a given level of accuracy would yield similar results, and thus, it was not necessary to consider every one of them.

As DFT options in Jaguar, we choose "medium" grid density for OPT and "fine" grid density for SPE calculations. Energy and RMS density matrix change convergence criteria are set to the default values of $5.0 \times 10^{-5}$ Hartree and $5.0 \times 10^{-6}$, respectively. The default direct inversion in the iterative subspace (DIIS, iconv $=1$ ) is employed as the convergence scheme. For OPT, the option iaccg $=2$ is employed as it increases the speed of computations by using Jaguar's mixed pseudospectral grids with default cutoffs. For SPE calculations, we set iacc $=2$ that corresponds to accurate cutoffs, and use pseudospectral grids. To treat solvated molecular systems in water, we use the standard PBF solver (isolv $=2$ ) with water as the solvent. ${ }^{39,43}$ The calculations are performed with LACVP ${ }^{* *+}$ basis set with polarization and diffuse functions. ${ }^{68,69}$ The LACVP basis set is chosen here because it includes an effective core potential (ECP), which represents the effect of the core electrons in a parametrized form. The use of ECPs speeds up calculations on compounds that contain heavy elements. For the elements from $\mathrm{H}$ to Ar, LACVP and the widely employed 6-31G are essentially indistinguishable when evaluating ground state properties. The quinone molecules considered in this work contain the elements $\mathrm{C}, \mathrm{H}, \mathrm{O}, \mathrm{N}, \mathrm{S}, \mathrm{F}$ and $\mathrm{Cl}$, and thus, the use of $\mathrm{LACVP}^{* *++}$ basis set in this work is consistent with the use of $6-31 \mathrm{G}^{* *++}$ basis set. More information on LACVP basis sets can be found in the Jaguar User Manual. ${ }^{69}$ For the geometries that have been obtained from FF, SEQM and DFTB optimizations, the DFT level SPEs are computed in the gas phase, and subsequently in the aqueous phase using only 
the PBE, B3LYP, and M08-HX functionals, as they are well accepted in the community but also span a wide range of ways for accounting for the exchange-correlation effects.

\subsection{Calibration data and performance metrics}

We collected redox potential data from 43 quinone redox couples in acidic aqueous solution. ${ }^{15,22,29,70}$ In consideration of prediction accuracy and universality for the calibration models, the selection of available experimental data has been substantially expanded within various quinone molecules, rather than using monotonous structural patterns. Compounds decorated with various chemical functional groups can show improved redox properties as well as charge/discharge capacity when compared to their undecorated counterparts. These selected molecules cover both quinone cores and their functionalized derivatives with multiple substituted groups including $-\mathrm{SO}_{3} \mathrm{H},-\mathrm{COOH},-\mathrm{OH},-\mathrm{CH}_{3},-$ $\mathrm{F}$ and $-\mathrm{Cl}$ (see Supporting Information Table S1). The collected data spans a broad set of experimental redox potentials between -0.084 and $1.21 \mathrm{~V}$. The redox couples are chosen consistently from measurements that were performed under similar experimental conditions, such as $T=298.15 \mathrm{~K}, \mathrm{pH}$ $=0$, and highly conducting salts.

In this work, the correlations between experiments and calculations are expressed in terms of two commonly used coefficients, namely the coefficient of determination $\left(\mathrm{R}^{2}\right)$ and root-mean-square error (RMSE). $\mathrm{R}^{2}$ and RMSE in this work are calculated using the definitions from the software Originlab, in which RMSE is defined as $\sqrt{\frac{\sum_{i=1}^{n}\left(y_{i}-\hat{y}_{i}\right)^{2}}{n-1}}$, where $y_{i}$ is the experimental measurement made at the $\mathrm{i}^{\text {th }}$ $x$-value in the data set, $\hat{y}_{i}$ is the predicted response for the measurement. The $x$-value in this study refers to calculated reaction energy differences, $\Delta E_{\mathrm{rxn}}$, as described earlier.

\section{Results and discussions}

\subsection{Comparison of the DFT methods}


DFT is the highest level of theory considered in this work with regards to accuracy in the prediction of total energies. Therefore, we begin with a discussion of the performance of the various DFT functionals, with an aim to use them as performance benchmarks for the lower-level methods. At first, we briefly discuss the performance of $\Delta U_{\mathrm{rxn}}$ and $\Delta G_{\mathrm{rxn}}^{\mathrm{o}}$ as descriptors for predicting redox potentials. For this purpose, DFT energy calculations using the PBE functional are performed first for optimizing geometry in the gas phase, and then, for calculating single point energy in the implicit aqueous phase. The calibration performance of both $\Delta U_{\mathrm{rxn}}\left(\mathrm{RMSE}=0.049 \mathrm{~V}, \mathrm{R}^{2}=0.978\right)$ and $\Delta G_{\mathrm{rxn}}^{\mathrm{o}}(\mathrm{RMSE}=0.048$ $\mathrm{V}, \mathrm{R}^{2}=0.979$ ) is very similar, as shown in Fig. $\mathrm{S} 1$ in the Supporting Information. As will be demonstrated in the following discussions, inclusion of ZPE (in $\Delta U_{\mathrm{rxn}}$ ) as well as entropic effects (in $\left.\Delta G_{\mathrm{rxn}}^{\mathrm{o}}\right)$ is only marginally better than considering only $\Delta E_{\mathrm{rxn}}\left(\mathrm{RMSE}=0.051 \mathrm{~V}, \mathrm{R}^{2}=0.977\right)$. In this work, we consider that the effect of including these terms is not significant enough from a HTCS perspective. Therefore, all the following discussions in this work consider only $\Delta E_{\text {rxn }}$ as the descriptor. First, we discuss the linear calibration of three representative DFT functionals, namely: PBE (Fig. 2a), B3LYP (Fig. 2b), and M08-HX (Fig. 2c). As shown in the legend in Fig. 2, for each of these three functionals we further used three kinds of DFT calculated reaction energies $\Delta E_{\mathrm{rxn}}=\Delta E_{\mathrm{rxn}}^{\mathrm{DFT}}$, against the experimentally measured redox potentials $\left(E_{\exp }^{o}\right)$ as follows: $(1)$ with OPT in gas phase without calculation of the SPE in SOL, (2) with OPT in gas phase and the following SPE in SOL, and (3) with both OPT and SPE in SOL.

On comparing the RMSE and $\mathrm{R}^{2}$, the following observations are made:

(1) When using $\Delta E_{\mathrm{rxn}}^{\mathrm{DFT}}$ from only gas phase optimized geometry and SPE, PBE is the worst performing functional with $\mathrm{RMSE}=0.072 \mathrm{~V}, \mathrm{R}^{2}=0.954$. This indicates that at the most basic DFT level, it is possible to predict $E_{\exp }^{\mathrm{o}}$ for quinone-based molecules within the range of common experimental errors $(\sim 0.1 \mathrm{~V})$. 
(2) Upon the inclusion of solvation effects on the gas phase geometry using the implicit model, all three functionals show a considerable decrease in their RMSE values. The decrease in error is highest for PBE ( 30\%) and lowest for M08-HX ( 23\%).

(3) Remarkably for all three functionals, full geometry optimization and energy calculation in the implicit solvation model yields slightly worse results than their counterparts in which geometry is optimized in the gas phase. The RMSE values are found to increase by $0.002 \sim 0.004 \mathrm{~V}$, indicating that there is no real added value of performing geometry optimizations with implicit solvation, not to mention are computationally more demanding.

Based on the findings above, we evaluate the performance of eight other DFT functionals without considering geometry optimization in the implicit aqueous phase. In Fig. $2 \mathrm{~d}$ and Supporting Information Fig. S2, a summary of the performance of all DFT functionals considered in this work is presented in the form of bar plots for $\mathrm{R}^{2}$ and RMSE, respectively. When compared under the same set of approximations, it can be observed that, with the exception of LDA, all other functionals have relatively similar performance. The functionals PBE0/PBE0-D3, HSE06 and M08-HX have all very similar performance $\left(\mathrm{R}^{2} \sim 0.984\right.$, RMSE $\left.\sim 0.043 \mathrm{~V}\right)$ when using implicit solvation on a gas phase optimized geometry, which is followed by the hybrid functional B3LYP/B3LYP-D3 and then the GGA functionals (PBE/PBE-D3 and BLYP/BLYP-D3). The addition of D3-dispersion corrections makes hardly any difference on either of the hybrid or the GGA functional calculated results. For all further comparisons in this work, we choose PBE as the benchmark DFT functional amongst the compared DFT functionals, as it offers the best compromise between prediction accuracy and computational expense. It must be noted that in this work, the different DFT functionals are compared purely on the basis of their performance in predicting measured potentials. Functionals constructed with higher degrees of empiricism, such as the Minnesota Density Functionals, ${ }^{67}$ are aimed at producing better values of a chosen set of physically observable properties. In this regard, it is not surprising that the M08-HX functional performs the best amongst the compared functionals, as it is very heavily 
parametrized to show high performance for thermochemistry. However, it must be kept in mind that such heavily parametrized functionals tend to produce less accurate electron densities than the ones with little to no empiricism in their design, such as the PBE functional. ${ }^{62}$

(a)
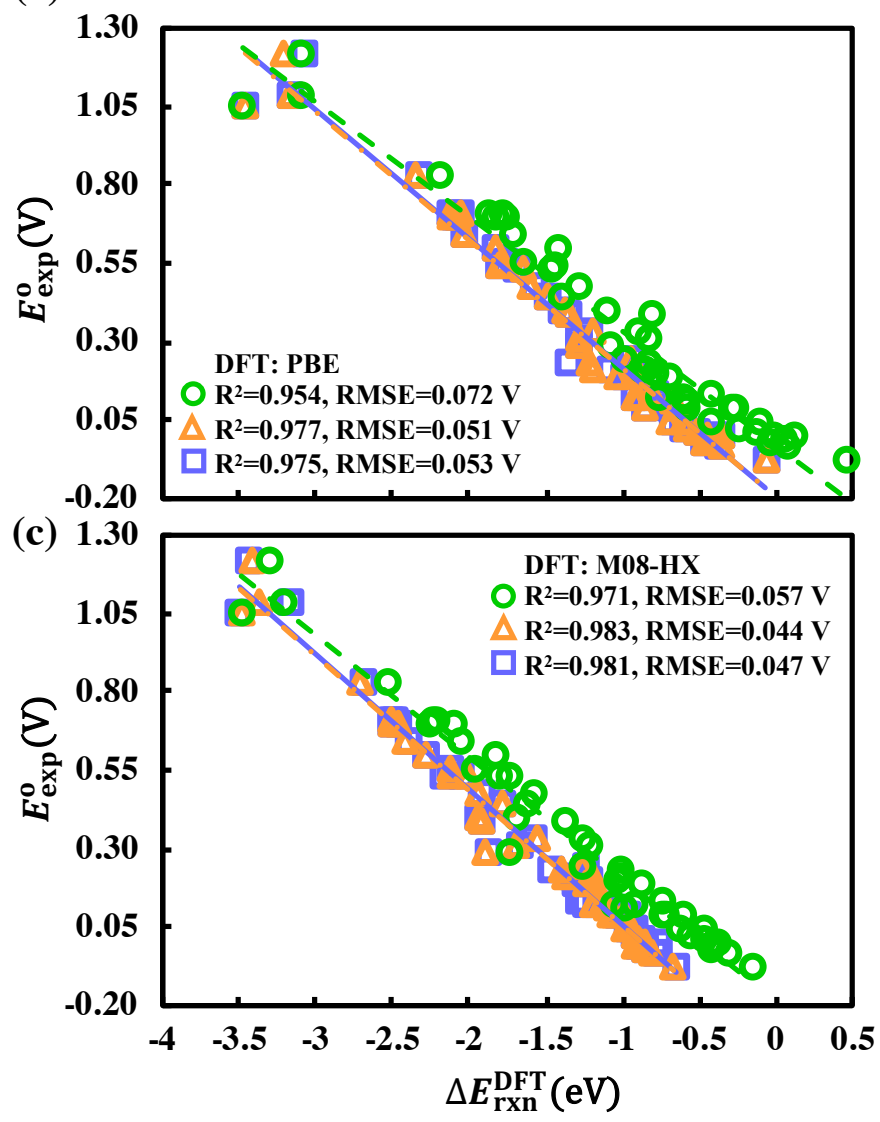

(b)
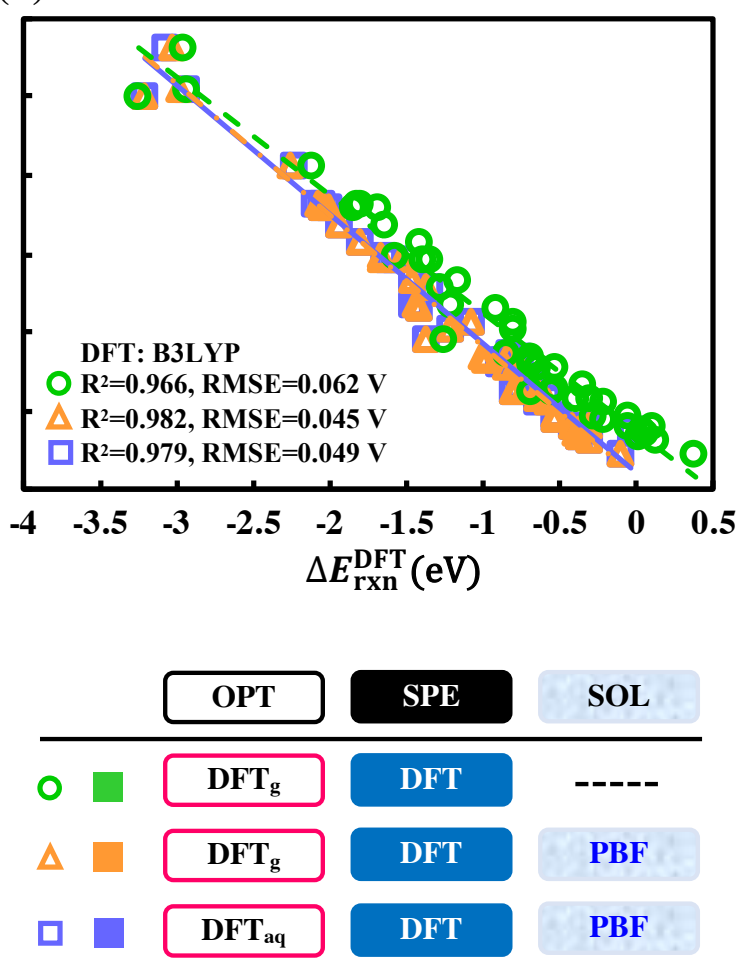

(d)

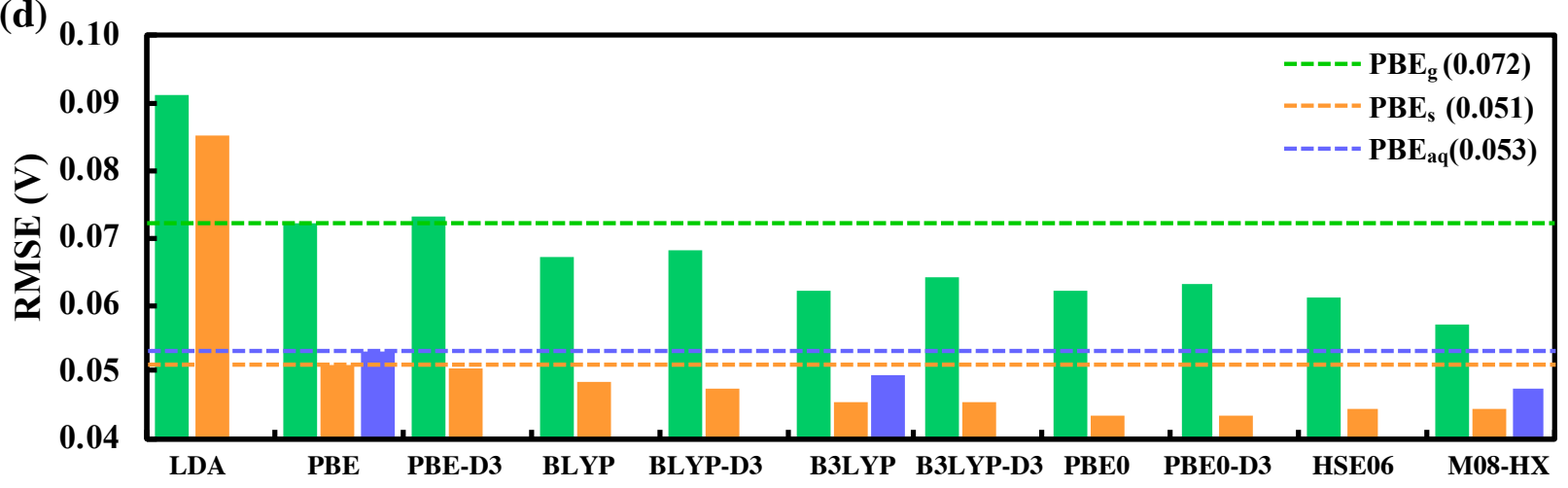

Fig. 2 Performance comparison of different DFT exchange-correlation functionals for prediction of experimentally measured redox potentials, $E_{\mathrm{exp}}^{\mathrm{o}}$. The scatter plots (a), (b) and (c) show the linear correlation of the DFT calculated energy difference, $\Delta E_{\text {rxn }}^{\mathrm{DFT}}$, versus $E_{\text {exp }}^{\mathrm{o}}$, for three representative 
functionals: (a) PBE, (b) B3LYP and (c) M08-HX. The bar plot (d) shows the RMSE values for all the functionals considered in this work. In all subplots of this figure, the color green represents both OPT and SPE in gas phase, the color orange represents OPT in gas phase followed by SPE with SOL (i.e., including implicit aqueous solvation) and color blue represents both OPT and SPE with SOL. The same information is tabulated in the legend. The horizontal dashed green line represents the $\mathrm{PBE}_{\mathrm{g}}$ $\left(\mathrm{RMSE}=0.072 \mathrm{~V}, \mathrm{R}^{2}=0.954\right)$ benchmark, the horizontal dashed blue line represents the PBE $\mathrm{aq}$ $\left(\mathrm{RMSE}=0.053 \mathrm{~V}, \mathrm{R}^{2}=0.975\right)$ benchmark, and the horizontal dashed orange line represents the $\mathrm{PBE}_{\mathrm{s}}$ $\left(\mathrm{RMSE}=0.051 \mathrm{~V}, \mathrm{R}^{2}=0.977\right)$ benchmark.

Discussion: It can be noted that using DFT with LDA density functional is clearly not as good as the GGA- and hybrid-level density functionals. Additionally, there is a significant impact on prediction accuracy due to the inclusion of the effect of implicit solvation in the calculation of $\Delta E_{\mathrm{rxn}}^{\mathrm{DFT}}$. Such an effect can be attributed to a better accounting of the -OH groups' interaction with the surrounding aqueous environment in the hydroquinone products. ${ }^{34}$ Surprisingly, optimizing geometry with implicit solvation slightly worsens the prediction accuracy. This observation can be attributed to multiple factors. First, it is possible that the PBF solvation model is not accurate enough to improve the gas phase geometry. Secondly, there might be a serendipitous cancellation of errors when using the gas phase geometry that is affected by the changes in the geometry due to the implicit solvation model in use. Additionally, $\Delta E_{\mathrm{rxn}}$ is used as an approximation for $\Delta G_{\mathrm{rxn}}^{\mathrm{o}}$, and accounting for the ignored pressure-volume and entropy terms from Eq. (4) might result in better prediction accuracy when optimizing geometry implicit solvation. In the work of Kim et al., ${ }^{34}$ it was shown that the reduction potentials of Anthraquinones in acidic aqueous solutions are strongly influenced by specific interactions with molecules in the solvent environment. In aqueous solution, they found that using DFT ( $\omega$ B97X-D/6-31G*) with implicit solvation (PCM(Bondi)) for the geometry optimization yields good results, except for the redox couples that have strong intramolecular hydrogen bond interactions. 
They evaluated a total of 19 Anthraquinones and identified a mean absolute deviation (MAD) of 0.194 V for three outliers that showed strong intramolecular hydrogen bond interactions. This value was more than five times the MAD value of the remaining 16 redox couples $(0.037 \mathrm{~V})$. Further, they showed that QM/MM calculations (with the TIP3P force field for explicit water molecules) can alleviate the overestimation and lead to a more balanced treatment of the solute-solvent interactions. Accordingly, using a QM/MM model, the correlation between theory and experiment had a MAD of $0.033 \mathrm{~V}$. In the current work, we performed a similar analysis of MAD values in our calibration set with 43 molecule pairs. We found that there are only 12 molecules, with IDs: 1, 2, 3, 4, 5, 6, 8, 9, 16, 35, 37 and 39 from Table S1 in the Supporting Information, without any possibility of strong intramolecular hydrogen bond interactions due to the neighboring positions found in the hydroquinone versions of the molecules. Surprisingly, we found that when using implicit aqueous solvation during geometry optimization, the MAD in prediction of redox potentials was $0.039 \mathrm{~V}$ for the 12 molecules and $0.037 \mathrm{~V}$ for the remaining 31 molecules. We note that these MADs are very similar and the difference between the two groups is only in the third decimal digit. Therefore, we cannot confirm that the explanation provided by Kim et al. also applies to the methods used in this work. At the same time, it must be noted that Kim et al. used only Anthraquinones (3-ring molecules) for their analysis, whereas this work considers a wide variety of quinone molecules (from 1 to 3 rings), including those with the $\mathrm{C}=\mathrm{O}$ groups at the 1,2 positions on the compounds. Further, Kim et al. employed the PCM (Bondi) implicit solvation model, which is different from the PBF model used in the current work. These differences, as well as the difference in the calibration data, make it hard to ascertain the exact origin of the disparities between this work and the work of Kim et al.

Another important aspect of the calibration of molecules that needs to be considered is the effect of ionization of sulfonic acid groups, as they are prone to dissociation in aqueous media. In the calibration set of 43 molecule pairs used in the current work, there are 18 molecules, with IDs: 7, 8, 9, 10, 11, 12, 13, 30, 31, 32, 33, 34, 35, 36, 37, 41, 42 and 43 from Table S1 in the Supporting Information, which 
contain $-\mathrm{SO}_{3} \mathrm{H}$ groups. In the framework of the best performing scheme, i.e. $\mathrm{PBE}_{\mathrm{s}}$, we found that the MAD values for these 18 molecules is $0.047 \mathrm{~V}$, which is $\sim 50 \%$ higher than the MAD of the remaining 25 molecules $(0.032 \mathrm{~V})$. Clearly, the ionization of sulfonic groups has adverse effects on the prediction accuracy. Although the effect is not significant from the perspective of HTCS, we recommend the inclusion of explicit water molecules, such as in a QM/MM type of formalism, when highly accurate values of redox potentials of the quinones with sulfonic groups are demanded. ${ }^{34}$

The calibration equation for the prediction of redox potential vs SHE from energy difference of reaction corresponding to the $\mathrm{PBE}_{\mathrm{s}}(\equiv \mathrm{T})$ method is:

$E^{\mathrm{o}}=-0.409\left[\Delta E_{\mathrm{rxn}}^{\mathrm{DFT}}\right]-0.193$

The performance metrics of all the DFT functionals and their variations considered in this work can be found on the Supporting Information Table S2.

\subsection{Comparison of lower level methods: FF, SEQM and DFTB}

After establishing the effectiveness of DFT-based methods as a benchmark, we now turn to other, computationally less expensive methods of optimizing geometries and estimating energies of molecules. As summarized in Fig. 3 and Supporting Information Fig. S3, we employ different lower level methods such as FF, SEQM and DFTB for geometry optimization. Using these optimized structures, we calculate the $\Delta E_{\mathrm{rxn}}^{\mathrm{T}}$ from SPEs using the following three schemes:

I. SPE values are taken directly at the same lower level method after geometry optimization in the gas phase and in the aqueous phase. It must be noted that aqueous phase geometry optimization is performed only using FF and SEQM (but not DFTB, as explained in the Computational Workflow). 
II. SPE values are taken from gas phase DFT calculations using three different functionals (PBE, B3LYP and M08-HX), on the molecular geometries obtained through scheme (I).

III. SPE values are taken from DFT calculations with implicit solvation using three different functionals (PBE, B3LYP and M08-HX), on the molecular geometries obtained through scheme (I).

Several observations can be made on comparing the RMSE and $\mathrm{R}^{2}$ values across the various methodological combinations.

Comparisons within scheme (I): When comparing redox potential predictions from SPE values in scheme (I) to the PBEg benchmark $\left(\mathrm{R}^{2}=0.954, \mathrm{RMSE}=0.072 \mathrm{~V}\right)$, we make the following observations. Please note that the subscript ' $g$ ' represents gas phase and subscript ' $a q$ ' represents aqueous phase geometry optimization at a given level of theory.:

- In Fig. 3a, the gas phase (OPLS3eg: $\mathrm{R}^{2}=0.596$, RMSE $=0.213 \mathrm{~V}$ ) and aqueous phase (OPLS3 $\mathrm{e}_{\mathrm{aq}}: \mathrm{R}^{2}=0.060, \mathrm{RMSE}=0.325 \mathrm{~V}$ ) calculated FF SPE are far worse than PBEg. Just as observed for the case of DFT methods, aqueous phase FF geometry optimization yields worse results than its gas phase counterpart. Clearly the, internal energy predictions at the FF level are quite inaccurate.

- In Fig. 3a, the gas phase SEQM methods show significantly better performance compared to the FF method, and are close to the PBE $\mathrm{g}$ benchmark. Of note are the AM1 $\left(\mathrm{R}^{2}=0.899\right.$, RMSE $=0.107 \mathrm{~V})$ and $\mathrm{PM} 7_{\mathrm{g}}\left(\mathrm{R}^{2}=0.906, \mathrm{RMSE}=0.103 \mathrm{~V}\right)$ methods. The aqueous phase SEQM geometry optimization with the COSMO solvation model results in very similar prediction accuracy to their gas phase counterparts for both $\mathrm{AM}_{\mathrm{aq}}\left(\mathrm{R}^{2}=0.886, \mathrm{RMSE}=0.113 \mathrm{~V}\right)$ and $\mathrm{PM}_{\mathrm{aq}}\left(\mathrm{R}^{2}=0.915, \mathrm{RMSE}=0.098 \mathrm{~V}\right)$ SEQM methods.

- In Fig. 3a, the gas phase DFTB methods perform already as good as the PBE $\mathrm{g}$ benchmark with parameter sets DFTB-D3 $g\left(R^{2}=0.953\right.$, RMSE $\left.=0.072 \mathrm{~V}\right)$ and GFN1-XTB $\left(\mathrm{R}^{2}=0.944, \mathrm{RMSE}\right.$ $=0.079 \mathrm{~V}$ ). 
Comparisons within scheme (II): When comparing predictions from SPE values in scheme (II) to the corresponding PBEg benchmark $\left(\mathrm{R}^{2}=0.954\right.$, RMSE $\left.=0.072 \mathrm{~V}\right)$, we make the following observations:

- In Fig. 3b (solid bars), the performance of gas phase DFT calculations of SPE on gas phase FF geometries (OPLS3 $\mathrm{e}_{\mathrm{g}}: \mathrm{R}^{2}=0.947, \mathrm{RMSE}=0.077 \mathrm{~V}$ ) is significantly better than their counterparts from scheme (I). The same can be observed for the gas phase DFT calculations of SPE using aqueous phase FF geometries (OPLS3e $\left.\mathrm{eq}_{\mathrm{aq}}: \mathrm{R}^{2}=0.939, \mathrm{RMSE}=0.083 \mathrm{~V}\right)$. However, even after performing DFT calculations of SPE, OPLS3 $\mathrm{e}_{\mathrm{aq}}$ performs worse than OPLS3 $\mathrm{e}_{\mathrm{g}}$.

- In Fig. 3b (solid bars), the gas phase DFT calculations of SPE on gas phase SEQM geometries also show improved prediction accuracy with respect to their counterparts from scheme (a). The two best SEQM methods are AM1 $\left(\mathrm{R}^{2}=0.963, \mathrm{RMSE}=0.064 \mathrm{~V}\right)$ and PM7g $\left(\mathrm{R}^{2}=0.954\right.$, RMSE $=0.072 \mathrm{~V}$ ), with performance equaling the $\mathrm{PBE}_{\mathrm{g}}$ benchmark. Gas phase DFT calculations of SPE on aqueous phase SEQM geometries resulted in worse predictions for both $\mathrm{AM}_{\mathrm{aq}}\left(\mathrm{R}^{2}=0.956, \mathrm{RMSE}=0.070 \mathrm{~V}\right)$ and $\mathrm{PM} 7_{\mathrm{aq}}\left(\mathrm{R}^{2}=0.943, \mathrm{RMSE}=0.080 \mathrm{~V}\right)$, though they are still better with respect to their counterparts from scheme (I).

- In Fig. 3b (solid bars), the gas phase DFT calculations of SPE on gas phase DFTB geometries also show slightly improved prediction accuracy and are slightly better than the $\mathrm{PBE}_{\mathrm{g}}$ benchmark with parameter sets DFTB-D3g $\left(\mathrm{R}^{2}=0.960\right.$, RMSE $\left.=0.067 \mathrm{~V}\right)$ and GFN1-XTBg $\left(\mathrm{R}^{2}=0.949, \mathrm{RMSE}=0.075 \mathrm{~V}\right)$.

Comparisons within scheme (III): Upon including implicit solvation effects during DFT calculations of SPE, the performance of the lower level theories versus the corresponding $\mathrm{PBE}_{\mathrm{s}}$ benchmark $\left(\mathrm{R}^{2}=\right.$ $0.977, \mathrm{RMSE}=0.051 \mathrm{~V}$ ) can be described as follows (please note that the subscript $s$ represents gas phase geometry optimization but with implicit solvation included while calculating SPE with DFT):

- In Fig. 3b (dashed bars), the RMSE values are lower by $0.02 \mathrm{~V}$ for FF optimized geometries both from the gas $\left(\right.$ OPLS3 $\left.\mathrm{e}_{\mathrm{g}}: \mathrm{R}^{2}=0.969, \mathrm{RMSE}=0.059 \mathrm{~V}\right)$ and the aqueous phase $\left(\right.$ OPLS3 $\mathrm{e}_{\mathrm{aq}}$ : 
$\left.\mathrm{R}^{2}=0.964, \operatorname{RMSE}=0.063 \mathrm{~V}\right)$, in comparison to their counterparts from scheme (II). Surprisingly, the performance of these methods is found to be close to the $\mathrm{PBE}_{\mathrm{s}}$ benchmark. This indicates that even though the thermochemistry with FF obtained energies is not accurate (as observed in Scheme I), the geometries from FF for quinone molecules is good enough for performing DFT SPE calculations.

- In Fig. 3b (dashed bars), the prediction accuracy from SEQM optimized geometries is improved when compared to their counterparts in scheme (II). The two best SEQM methods are $A M 1_{g}\left(R^{2}=0.969, R M S E=0.059 \mathrm{~V}\right)$ and $P M 7 g\left(R^{2}=0.976, R M S E=0.051 \mathrm{~V}\right)$. Yet again, the predictions from aqueous phase SEQM geometries resulted in slightly worse predictions for both $\mathrm{AM} 1_{\mathrm{aq}}\left(\mathrm{R}^{2}=0.961, \mathrm{RMSE}=0.066 \mathrm{~V}\right)$ and $\mathrm{PM} 7_{\mathrm{aq}}\left(\mathrm{R}^{2}=0.962, \mathrm{RMSE}=0.065 \mathrm{~V}\right)$. The performance of these SEQM methods is interestingly close to the $\mathrm{PBE}_{\mathrm{s}}$ benchmark.

- In Fig. 3b (dashed bars), the prediction accuracy from DFTB optimized geometries improves when compared to their counterparts in scheme (II). Both sets of DFTB parameters, the DFTB$\mathrm{D} 3_{\mathrm{g}}\left(\mathrm{R}^{2}=0.978, \mathrm{RMSE}=0.049 \mathrm{~V}\right)$ and GFN1-XTB ${ }_{\mathrm{g}}\left(\mathrm{R}^{2}=0.977, \mathrm{RMSE}=0.051 \mathrm{~V}\right)$ perform better than the $\mathrm{PBE}_{\mathrm{s}}$ benchmark methods. 

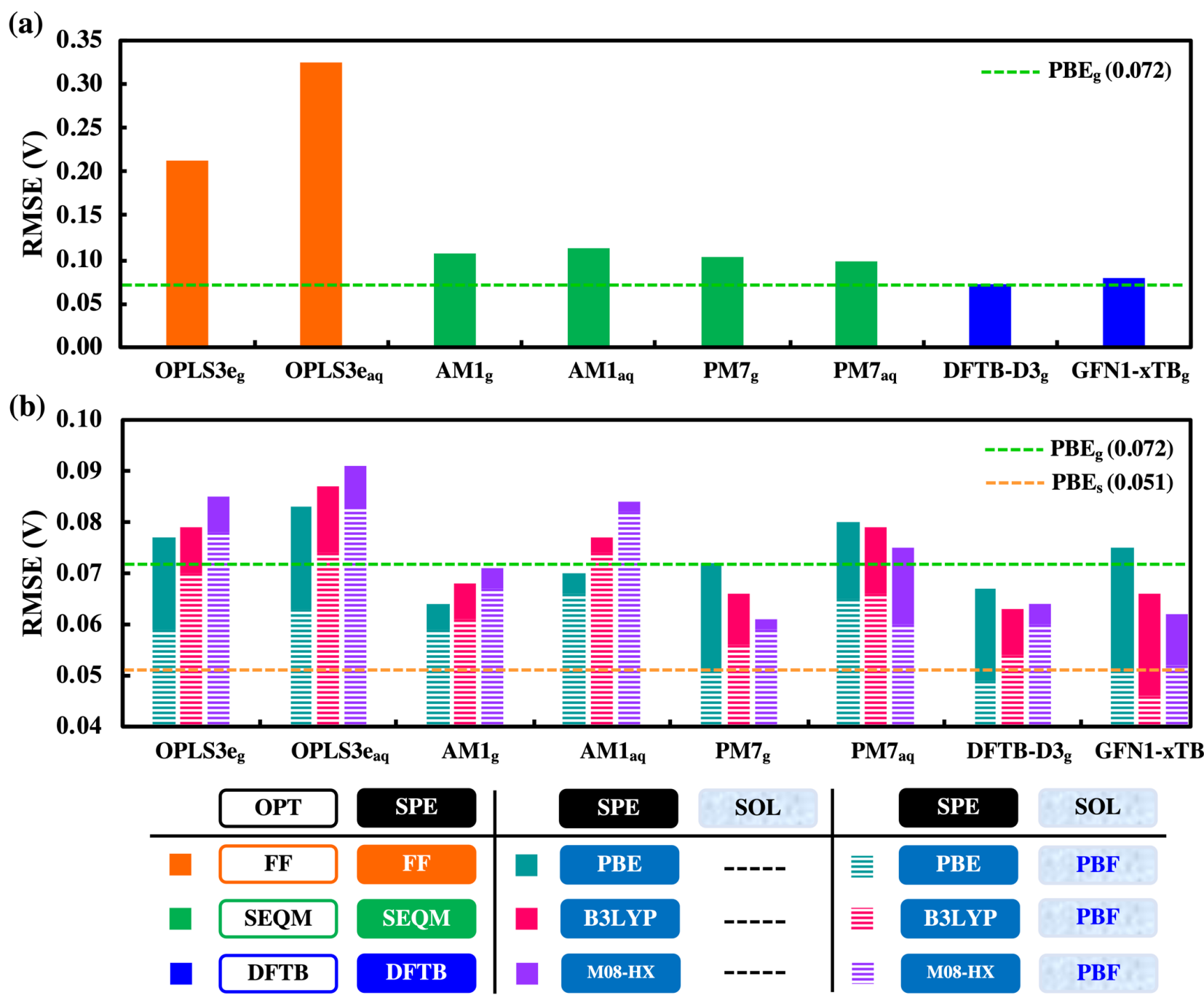

Fig. 3 Performance comparison of lower level methods: FF, SEQM and DFTB. (a) shows the RMSE for SPE values calculated at these three different levels of theory. Similarly, (b) show the RMSE for the DFT calculated SPE data on the geometries obtained from these three different levels of theory. In (b), the solid bars show the SPE results without the implicit solvation effect whereas the dashed bars show the results with the implicit solvation effects taken into account. The dashed green horizontal line represents the $\mathrm{PBE}_{\mathrm{g}}\left(\mathrm{R}^{2}=0.954, \mathrm{RMSE}=0.072 \mathrm{~V}\right)$ benchmark and the dashed orange horizontal line represents the $\mathrm{PBE}_{\mathrm{s}}\left(\mathrm{R}^{2}=0.977, \mathrm{RMSE}=0.051 \mathrm{~V}\right)$ benchmark.

Discussion: All the variations in computational methods that are used for geometry optimization and SPE calculation, with and without implicit solvation effects, are found to influence prediction accuracy 
to varying degrees. First, for all methods, the gas phase DFT calculation of SPE leads to significant improvements in prediction accuracy. This also implies that the computationally costly DFT geometry optimizations are hardly necessary for a first order screening of large numbers of candidate molecules. Instead, either of the SEQM or DFTB based methods can be employed for the task of gas-phase geometry optimizations. Secondly, SPE calculations employing the PBE density functional are generally better performing than with the computationally more expensive B3LYP and M08-HX functionals. Thirdly, for all the considered lower level methods, the inclusion of an implicit solvation model during the DFT calculation of SPE leads to improved prediction accuracies. Finally, these results confirm once again that the effect of geometry optimization in the aqueous phase is minimal and it often results in a slightly worse prediction accuracy. The calibration equation for the prediction of redox potential vs SHE from energy difference of reaction corresponding to the DFTB-D3 $\mathrm{g}_{\mathrm{g}}(\equiv \mathrm{T})$ method is:

$E^{\mathrm{o}}=-0.447\left[\Delta E_{\mathrm{rxn}}^{\mathrm{DFTB}}\right]-0.823$

The exact performance metrics of all the lower level methods and their variations considered in this work can be found in the Supporting Information Table S3-S9.

\subsection{Computation time vs. prediction accuracy}

In addition to determining the most accurate method for predicting the redox potentials of electroactive quinones, a major aim of the current study is to find out the methods that are most suited for both standalone and HTCS studies for which speed and accuracy of computations are highly desirable. This is particularly important when the robust DFT calculations become impractical for a study on a very large chemical space $\left(10^{3} \sim 10^{6}\right)$ of new candidate compounds. Thus, knowing the trade-offs between computational accuracy and expense is essential for an efficient screening of large chemical spaces of 
candidates. For a comparison of computation time of the various methods considered here, we selected a representative method for each level of theory, namely, OPLS3e (FF), PM7 (SEQM), DFTB-D3 (DFTB) and PBE (DFT). Next, noting that the geometry optimizations are usually the most computationally demanding processes, we optimized the geometries of all the molecules in the gas phase using these representative methods. We added the FF geometry optimization time to all other methods' calculation times, since we use it as the base method for performing all other geometry optimizations (as explained in the Computational Workflow). The averaged computation time calculated from five different runs, which showed no significant variation, is used to describe the relation between RMSE values from each of these methods versus their computational expense on a logarithmic scale, as shown in Fig. 4a. All of the benchmark simulations are performed on a single core of the Intel Core i9-9960X 3.10GHz CPU with Ubuntu 18.04 Bionic Beaver as the operating system. According to Fig. 4, DFTB-D3 is almost as accurate as the PBE method in predicting the redox

potentials, and it requires substantially ( $\sim 10^{3}$ times $)$ less computing time. Also noting that the DFTBD3 has previously been applied for the calculation of large systems at relatively low computational costs and with similar accuracies to that of the higher level (i.e. DFT-GGA functionals) methods,${ }^{71}$ we find that for the small redox compounds the DFTB-D3 method provides the best compromise between prediction accuracy and computational expense. Accordingly, for HTCS studies that are aimed work on extremely large chemical spaces of molecules, we suggest DFTB-D3 computations on OPLS3e optimized geometries as a feasible strategy to accelerate the virtual screening of compounds.

\subsection{The effects of geometry optimization at various levels of theory and implicit solvation}

For the set of methods considered in Section 3.3, we also first quantify the effect of gas phase DFT calculation of the SPE by using the geometries that are obtained from lower level theories. As shown in Fig. 4b (solid bars), the improvement in prediction accuracy, $\Delta^{\mathrm{e}} \mathrm{RMSE}$, is most significant for geometries optimized by OPLS3e $\left(\Delta^{\mathrm{e}} \mathrm{RMSE}=0.136 \mathrm{~V}\right)$, followed by PM7 $\left(\Delta^{\mathrm{e}} \mathrm{RMSE}=0.031 \mathrm{~V}\right)$, 
and lastly by DFTB-D3 $\left(\Delta^{\mathrm{e}}\right.$ RMSE $\left.=0.005 \mathrm{~V}\right)$. These results indicate that the DFTB based methods do not only predict the correct reaction energies but they as well predict the geometries that are close to that of DFT calculations. For the same set of methods, we also investigate the relationship between differences in molecular geometries and observed values of $\Delta^{\mathrm{e}} \mathrm{RMSE}$. In order to compare geometries from different methods with reference to the gas phase PBE geometry, we employed the structure superposition tool as implemented in the Schrödinger Materials Science Suite (version 2019-2) and obtained the average RMSD (in $\AA$ ) of all the 86 reactant and product molecules under various atomic constraints as shown in Table 2. The RMSD values have been calculated for all atoms, all nonhydrogen atoms, and carbon atoms in the rings.

Table 2. The difference in the optimized molecular geometry using various calculation methods with reference to the PBE (DFT) geometry under various atomic constraints. The average RMSD values for the 86 molecules are shown in units of $\AA$.

\begin{tabular}{|c|c|c|c|}
\hline Method & All atoms & Non - hydrogen & Only ring carbon \\
\hline DFTB-D3 & 0.135 & 0.102 & 0.043 \\
\hline PM7 (SEQM) & 0.132 & 0.098 & 0.050 \\
\hline OPLS3e (FF) & 0.148 & 0.120 & 0.052 \\
\hline
\end{tabular}

First, it can be observed that under all constraints OPLS3e has the largest average RMSD with respect to PBE, which is not surprising. Secondly, it is apparent that when considering all atoms or only heavy atoms, PM7 and DFTB-D3 are very similar in geometrical difference with regards to PBE. Thirdly, it is surprising to note that DFTB-D3, while the most accurate of the all lower level methods, does not necessarily provide the geometry closest to PBE (i.e. lowest average RMSD) under all types of atomic constraints. However, when considering only the carbon atoms in the rings, the DFTB-D3 produces structures that are closest to PBE. Given that the cyclic carbon atoms are a large fraction of the total number of atoms, it is possible that being able to represent the geometry of the rings accurately is what 
gives DFTB-D3 $\left(\Delta^{\mathrm{e}}\right.$ RMSE $\left.=0.005 \mathrm{~V}\right)$ a clear advantage over PM7 $\left(\Delta^{\mathrm{e}} \mathrm{RMSE}=0.031 \mathrm{~V}\right)$ and OPLS3e $\left(\Delta^{\mathrm{e}} \mathrm{RMSE}=0.136 \mathrm{~V}\right)$ in terms of prediction accuracy in redox potential.

Next, we quantify the improvement in prediction capability of the methods, with respect to gas phase DFT calculation of SPE, due to the inclusion of the implicit solvation, $\Delta^{\mathrm{s}} \mathrm{RMSE}$ (dashed bars), models. As shown in Fig. $4 \mathrm{~b}$, the $\Delta^{\mathrm{s}}$ RMSE is very similar at each level of theory. The lowering in RMSE values is $0.018,0.021,0.018$, and $0.021 \mathrm{~V}$ for OPLS3e, PM7, DFTB-D3, and PBE, respectively. These results imply that the amount of improvement due to the inclusion of the implicit solvation is independent of the source of geometry. Nevertheless, the improvement in prediction accuracy is evident for all levels of theory. These findings would be particularly useful, for instance, when building machine learning models for the prediction of solvation energies directly from the cheminformaticsbased descriptors and without the need for explicit knowledge of the 3D geometries of compounds.

(a)

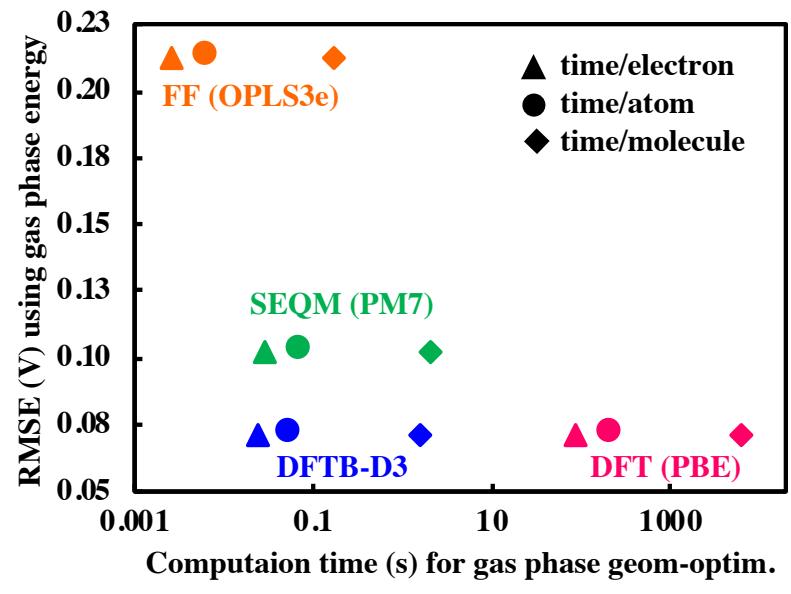

(b)

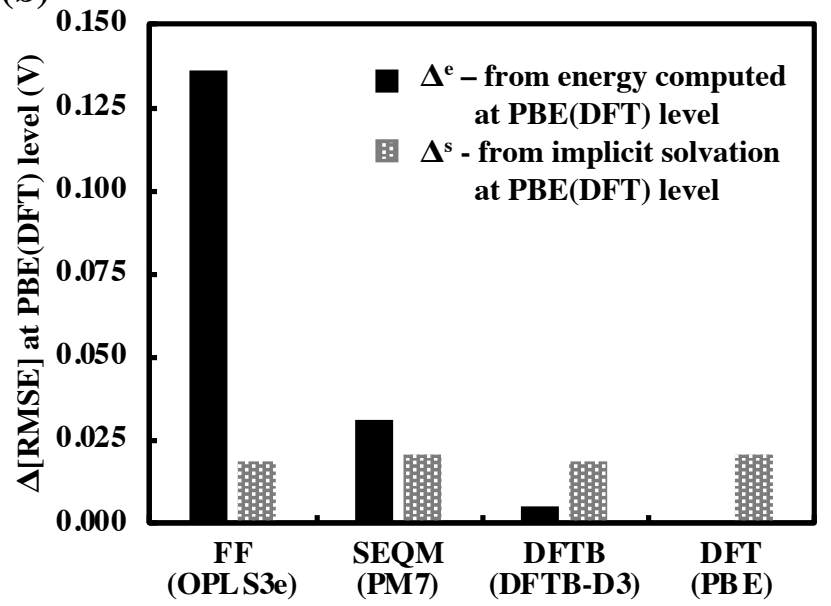

Fig. 4 (a) Variation of computation time for gas-phase geometry optimization $v s$ the corresponding RMSE values from the four representative methods of each level of theory, namely OPLS3e (FF), PM7 (SEQM), DFTB-D3 (DFTB), and PBE (DFT). The computation times are calculated by averaging over the total number of electrons, atoms, and molecules in the calibration set. The SPE values are taken directly after optimization at the corresponding level of theory. (b) Bar plot for lowering in RMSE values, $\Delta[\mathrm{RMSE}]$, due to gas phase DFT calculation of SPE and inclusion of 
implicit solvation. Solid bars, $\Delta^{\mathrm{e}}$, show the impact of DFT calculation of SPE on geometries from lower levels of theory. Dashed bars, $\Delta^{\mathrm{s}}$, show the impact of the including implicit solvation during DFT calculations of SPE.

\section{Conclusions}

We developed a systematic computational approach involving one FF, nine SEQM, two DFTB, and eleven DFT methods, as well as their combination with implicit solvation environments, to predict the redox potentials of 43 different experimentally measured quinone-based electroactive molecules collected from four sources. The reaction energy difference between the reactant and product is used as an approximation for the Gibbs free energy of proton-coupled electron transfer redox reactions. All the DFT functionals are found to perform well with RMSE values within common experimental errors $(\sim 0.1 \mathrm{~V})$. To evaluate the performance of the lower level methods, the PBE functional is chosen as an effective benchmark theoretical model, as it provides a good compromise between accuracy and computational cost when compared to other electronic structure methods. It is shown that the inclusion of implicit solvation during DFT calculation of SPE improves the prediction accuracy noticeably $(\sim 0.025 \mathrm{~V})$. Surprisingly, geometry optimization in the implicit aqueous phase is found to have no positive effect on prediction accuracy. The prediction accuracies of the lower level methods considered in this work are not as good as DFT, with the exception of DFTB that is found to be at par with DFT. DFT calculations of SPE using gas phase geometries obtained by FF and SEQM methods lead to significantly improved results, thus showing that the quantum chemical treatment of the electronic structure for the calculation of SPE is beneficial. This also implies that the expensive DFT geometry optimizations are not always necessary, for instance, in case of a first-order screening of millions of candidate molecules. The inclusion of implicit solvation during DFT calculation of SPE leads to a similar improvement across all the methods considered in this work. We recommend the DFTB-based methods as ideal approaches from the perspective of the trade-off between computational expense and 
prediction accuracy. Eq. (7) and Eq. (8) corresponding to the PBE $\mathrm{s}_{\mathrm{s}}$ and DFTB-D3g methods, respectively, can be applied directly to predict the redox potentials of quinone-based molecules vs SHE under the same set of approximations as used in the current work. Further, the strategy of optimizing geometry at a lower level of theory followed by single point energy calculation with DFT can be used to quickly generate a large amount of data which can be useful for building generative-predictive machine learning models for the discovery of useful electroactive molecules.

\section{Conflicts of interest}

There are no conflicts to declare.

\section{Acknowledgements}

This research received funding from the Netherlands Organisation for Scientific Research (NWO), through the COLORFLOW project partnership of DIFFER and Green Energy Storage, in the framework of the Materials for Sustainability programme and from the Ministry of Economic Affairs in the framework of the "PPS-Toeslagregeling" grant no 739.017.013. SE acknowledges funding from the initiative "Computational Sciences for Energy Research" of Shell and NWO grant no 15CSTT05. This work was sponsored by NWO Exact and Natural Sciences for the use of supercomputer facilities.

\section{Supporting Information}

Supplementary material associated with this article can be found in the online version. Contents: Explanation of the abbreviations; (Fig. S1) Performance of $\Delta U_{\text {rxn }}$ and $\Delta G_{\text {rxn }}^{\text {o }}$ (Fig. S2 - S3) Performance of different methods; (Table S1) A summary of quinone redox molecules considered in this study; (Tables S2 - S9) Performance comparisons of computational methods. 


\section{References}

1 Y. Ding, C. Zhang, L. Zhang, Y. Zhou and G. Yu, Chem. Soc. Rev., 2018, 47, 69-103.

2 V. Singh, S. Kim, J. Kang and H. R. Byon, Nano Res., 2019, 12, 1-14.

3 J. Noack, N. Roznyatovskaya, T. Herr and P. Fischer, Angew. Chemie - Int. Ed., 2015, 54, 9776-9809.

4 A. Z. Weber, M. M. Mench, J. P. Meyers, P. N. Ross, J. T. Gostick and Q. Liu, J. Appl. Electrochem., 2011, 41, 1137-1164.

5 G. Kear, A. A. Shah and F. C. Walsh, Int. J. Energy Res., 2012, 36, 1105-1120.

6 J. Winsberg, T. Hagemann, T. Janoschka, M. D. Hager and U. S. Schubert, Angew. Chemie Int. Ed., 2016, 55, 2-28.

7 C. Debruler, B. Hu, J. Moss, J. Luo and T. L. Liu, ACS Energy Lett., 2018, 3, 663-668.

8 W. Liu, Y. Liu, H. Zhang, C. Xie, L. Shi, Y. G. Zhou and X. Li, Chem. Commun., 2019, 55, 4801-4804.

9 K. Lin, R. Gómez-Bombarelli, E. S. Beh, L. Tong, Q. Chen, A. Valle, A. Aspuru-Guzik, M. J. Aziz and R. G. Gordon, Nat. Energy, 2016, 1, 16102.

10 W. Lee, B. W. Kwon and Y. Kwon, ACS Appl. Mater. Interfaces, 2018, 10, 36882-36891.

11 A. Hollas, X. Wei, V. Murugesan, Z. Nie, B. Li, D. Reed, J. Liu, V. Sprenkle and W. Wang, Nat. Energy, 2018, 3, 508-514.

12 J. Winsberg, C. Stolze, S. Muench, F. Liedl, M. D. Hager and U. S. Schubert, ACS Energy Lett., 2016, 1, 976-980.

13 A. Orita, M. G. Verde, M. Sakai and Y. S. Meng, J. Power Sources, 2016, 321, 126-134.

14 B. Huskinson, M. P. Marshak, C. Suh, S. Er, M. R. Gerhardt, C. J. Galvin, X. Chen, A. Aspuru-Guzik, R. G. Gordon and M. J. Aziz, Nature, 2014, 505, 195-198. M. R. Gerhardt, L. Tong, R. Gómez-Bombarelli, Q. Chen, M. P. Marshak, C. J. Galvin, A. Aspuru-Guzik, R. G. Gordon and M. J. Aziz, Adv. Energy Mater., 2017, 7, 1601488. 
16 Y. Liu, S. Lu, S. Chen, H. Wang, J. Zhang and Y. Xiang, ACS Appl. Energy Mater., 2019, 2, 2469-2474.

L. Tong, Y. Jing, R. G. Gordon and M. J. Aziz, ACS Appl. Energy Mater., 2019, 2, 40164021.

18 S. Jin, Y. Jing, D. G. Kwabi, Y. Ji, L. Tong, D. De Porcellinis, M.-A. Goulet, D. A. Pollack, R. G. Gordon and M. J. Aziz, ACS Energy Lett., 2019, 4, 1342-1348.

19 L. Li, Q. Zhao, Z. Luo, Y. Lu, H. Ma, J. Hu, Y. Li, J. Chen, L. Liu and W. Huang, Sci. Adv., 2018, 4, eaao1761.

20 C. Wang, Z. Yang, Y. Wang, P. Zhao, W. Yan, G. Zhu, L. Ma, B. Yu, L. Wang, G. Li, J. Liu and Z. Jin, ACS Energy Lett., 2018, 3, 2404-2409.

21 B. Lee, Y. Ko, G. Kwon, S. Lee, K. Ku, J. Kim and K. Kang, Joule, 2018, 2, 61-75.

22 K. Wedege, E. Dražević, D. Konya and A. Bentien, Sci. Rep., 2016, 6, 1-13.

23 M.-A. Goulet, Z. Yang, E. S. Beh, A. Aspuru-Guzik, L. Tong, M. J. Aziz, R. G. Gordon, D. De Porcellinis and D. P. Tabor, Adv. Energy Mater., 2017, 8, 1702056.

24 Y. Ji, M. A. Goulet, D. A. Pollack, D. G. Kwabi, S. Jin, D. De Porcellinis, E. F. Kerr, R. G. Gordon and M. J. Aziz, Adv. Energy Mater., 2019, 1900039, 1-7.

25 B. Yang, S. R. Narayanan, L. Hoober-Burkhardt, F. Wang and G. K. Surya Prakash, J. Electrochem. Soc., 2014, 161, A1371-A1380.

26 M. C. Sorkun, A. Khetan and S. Er, Sci. data, 2019, 6, 143.

27 L. Cheng, R. S. Assary, X. Qu, A. Jain, S. P. Ong, N. N. Rajput, K. Persson and L. A. Curtiss, J. Phys. Chem. Lett., 2015, 6, 283-291.

28 S. Er, C. Suh, M. P. Marshak and A. Aspuru-Guzik, Chem. Sci., 2015, 6, 885-893.

29 D. P. Tabor, R. Gómez-Bombarelli, L. Tong, R. G. Gordon, M. J. Aziz and A. Aspuru-Guzik, J. Mater. Chem. A, 2019, 7, 12833-12841. 

Soc., 2016, 138, 15903-15910.

31 A. Jinich, B. Sanchez-Lengeling, H. Ren, R. Harman and A. Aspuru-Guzik, ACS Cent. Sci., $2019,5,1199-1210$.

32 J. P. Janet, S. Ramesh, C. Duan and H. J. Kulik, ACS Cent. Sci., 2020, 6, 513-524.

33 J. Hachmann, R. Olivares-Amaya, A. Jinich, A. L. Appleton, M. A. Blood-Forsythe, L. R. Seress, C. Román-Salgado, K. Trepte, S. Atahan-Evrenk, S. Er, S. Shrestha, R. Mondal, A. Sokolov, Z. Bao and A. Aspuru-Guzik, Energy Environ. Sci., 2014, 7, 698-704. H. Kim, T. Goodson and P. M. Zimmerman, J. Phys. Chem. C, 2016, 120, 22235-22247.

35 A. F. Oliveira, P. Philipsen and T. Heine, J. Chem. Theory Comput., 2015, 11, 5209-5218.

36 M. Korth, Phys. Chem. Chem. Phys., 2014, 16, 7919-7926.

37 T. Husch, D. Yilmazer and M. Korth, Phys. Chem. Chem. Phys., 2015, 17, 3394-3401.

38 A. S. Christensen, T. Kubař, Q. Cui and M. Elstner, Chem. Rev., 2016, 116, 5301-5337.

39 A. D. Bochevarov, E. Harder, T. F. Hughes, J. R. Greenwood, D. A. Braden, D. M. Philipp, D. Rinaldo, M. D. Halls, J. Zhang and R. A. Friesner, Int. J. Quantum Chem., 2013, 113, 2110 2142.

40 G. Te Velde, F. M. Bickelhaupt, E. J. Baerends, C. Fonseca Guerra, S. J. A. van Gisbergen, J. G. Snijders and T. Ziegler, J. Comput. Chem., 2001, 22, 931-967.

41 D. Weininger, J. Chem. Inf. Comput. Sci., 1988, 1, 31-36.

42 K. Roos, C. Wu, W. Damm, M. Reboul, J. M. Stevenson, C. Lu, M. K. Dahlgren, S. Mondal, W. Chen, L. Wang, R. Abel, R. A. Friesner and E. D. Harder, J. Chem. Theory Comput., 2019, 15, $1863-1874$. 
44 M. J. S. Dewar, E. G. Zoebisch, E. F. Healy and J. J. P. Stewart, J. Am. Chem. Soc., 1985, 107, 3902-3909.

45

46

47

48

49 1101-1111.

52

53 355, 49-52.

M. J. S. Dewar and W. Thiel, J. Am. Chem. Soc., 1977, 99, 4899-4907.

W. Thiel and A. A. Voityuk, J. Phys. Chem., 1996, 100, 616-626.

J. J. P. Stewart, J. Comput. Chem., 1989, 10, 221-264.

J. J. P. Stewart, J. Mol. Model., 2007, 13, 1173-1213.

Y. Jeanvoine and R. Spezia, Theor. Chem. Acc., 2019, 138, 1-13.

J. J. P. Stewart, J. Mol. Model., 2013, 19, 1-32.

G. B. Rocha, R. O. Freire, A. M. Simas and J. J. P. Stewart, J. Comput. Chem., 2006, 27,

A. Klamt and F. Eckert, Fluid Phase Equilib., 2000, 172, 43-72.

Y. Chu, X. Zhang, M. Hillestad and X. He, Fluid Phase Equilib., 2018, 475, 25-36.

J. G. Brandenburg and S. Grimme, J. Phys. Chem. Lett., 2014, 5, 1785-1789.

S. Grimme, C. Bannwarth and P. Shushkov, J. Chem. Theory Comput., 2017, 13, 1989-2009.

J. Koopman and S. Grimme, ACS Omega, 2019, 4, 15120-15133.

S. H. Vosko, L. Wilk and M. Nusair, Can. J. Phys., 1980, 58, 1200-1211.

N. Mardirossian and M. Head-Gordon, Mol. Phys., 2017, 115, 2315-2372.

M. Swart, Chem. Phys. Lett., 2013, 580, 166-171.

W. Hujo and S. Grimme, Phys. Chem. Chem. Phys., 2011, 13, 13942-13950.

J. P. Perdew, K. Burke and M. Ernzerhof, Phys. Rev. Lett., 1996, 78, 3865-3868.

M. G. Medvedev, I. S. Bushmarinov, J. Sun, J. P. Perdew and K. A. Lyssenko, Science., 2017,

P. J. Stephens, F. J. Devlin, C. F. Chabalowski and M. J. Frisch, J. Phys. Chem., 1994, 98, $11623-11627$. 
64 M. Sánchez-Castellanos, M. M. Flores-Leonar, Z. Mata-Pinzón, H. G. Laguna, K. M. GarciáRuiz, S. S. Rozenel, V. M. Ugalde-Saldívar, R. Moreno-Esparza, J. J. H. Pijpers and C. Amador-Bedolla, Phys. Chem. Chem. Phys., 2019, 21, 15823-15832.

65 C. Adamo and V. Barone, Chem. Phys., 2001, 110, 6158-6170.

66 A. V. Krukau, O. A. Vydrov, A. F. Izmaylov and G. E. Scuseria, J. Chem. Phys., 2006, 125, 224106.

67 N. Mardirossian and M. Head-Gordon, J. Chem. Theory Comput., 2016, 12, 4303-4325.

68 P. J. Hay and W. R. Wadt, J. Chem. Phys., 1985, 82, 299-310.

69 Jaguar, version 10.5. Schrödinger, Inc., New York, NY, 2019

70 J. R. Tobias Johnsson Wass, E. Ahlberg, I. Panas and D. J. Schiffrin, J. Phys. Chem. A, 2006, 110, 2005-2020.

71 G. Zheng, H. A. Witek, P. Bobadova-Parvanova, S. Irle, D. G. Musaev, R. Prabhakar, K. Morokuma, M. Lundberg, M. Elstner, C. Köhler and T. Frauenheim, J. Chem. Theory Comput., 2007, 3, 1349-1367. 